\title{
Guidance Stabilization of Satellites Using the Geomagnetic Field
}

\author{
Francisco Miranda ${ }^{1,2}$ \\ ${ }^{1}$ Center for Research and Development in Mathematics and Applications, University of Aveiro, Portugal \\ ${ }^{2}$ School of Technology and Management, Polytechnic Institute of Viana do Castelo, Avenida do Atlântico, \\ 4900-348 Viana do Castelo, Portugal \\ Correspondence should be addressed to Francisco Miranda, fmiranda@estg.ipvc.pt
}

Received 21 October 2011; Revised 13 January 2012; Accepted 18 January 2012

Academic Editor: Kenneth M. Sobel

Copyright () 2012 Francisco Miranda. This is an open access article distributed under the Creative Commons Attribution License, which permits unrestricted use, distribution, and reproduction in any medium, provided the original work is properly cited.

In the last years the small satellites have played an important role in the technological development. The attractive short period of design and low cost of them and the capacity to solve problems that are usually considered as problems to big and expensive spacecrafts lead us to study the control problem of these satellites. Active three-axis magnetic attitude stabilization of a low Earth orbit satellite is considered in this work. The control is created by interaction between the magnetic moment generated by magnetorquers mounted on the satellite body and the geomagnetic field. This problem is quite complex and difficult to solve. To overcome this difficulty guidance control is considered, where we use $\varepsilon$-strategies introduced by Pontryagin in the frame of differential games theory. Qualitative analysis and results of numerical simulation are presented.

\section{Introduction}

The problem of attitude control of spacecraft has been widely studied in the last years. If the spacecraft is equipped with three independent actuators, a complete solution to the set point and tracking control problems is available. This problem was solved by different ways (see, e.g., [1-4]). If only two independent actuators are available, as discussed in detail in [5], the problem of attitude regulation is not solvable by means of continuous time-invariant control laws, whereas a time-varying control law, achieving local asymptotic nonexponential stability, was proposed in [6]. Since magnetorquers are relatively reliable, lightweight, and energy efficient, they have become attractive for small and inexpensive satellites, but the above results are not directly applicable if the satellite is equipped with these magnetic coils as actuators. Such actuators operate on the basis of the interaction between the magnetic field generated by magnetic coils installed on the satellite body and the magnetic field of the Earth. This implies a spacecraft control significantly different. There are two classical uses of magnetic torque rods in attitude control. One is for momentum management of wheel-based systems [7]. The other is for angular momentum and nutation control of spinning [8], momentum-biased [9], and dual-spin spacecraft [10]. The present study is one of a growing number that considers active three-axis magnetic attitude stabilization of a low Earth orbit satellite considering a nontilted dipole model for the geomagnetic field. Magnetic coils have been extensively used since the early sixties as a simple and reliable technology to implement attitude control actuators in low Earth orbit satellites (see, e.g., [11-16] and the survey [17] and the references therein). The main difficulty in the implementation of this active three-axis magnetic system consists in the fact that the control torque can only be generated perpendicular to the geomagnetic field vector, which results in a time-varying nonlinear system. To solve this problem the most natural is to use the Lyapunov functions method combined with the Krasovskii-LaSalle theorem [18]. The Lyapunov function is used to construct a stabilizer depending on current time and position of the system. Then, the Krasovskii-LaSalle theorem is applied to prove the asymptotic stability of the equilibrium position. However, a rigorous verification of the Krasovskii-LaSalle theorem conditions faces serious technical difficulties which can hardly be overcome even with the help of systems for symbolic computations. Other techniques were presented (see, e.g., $[19,20]$ ). In [19], Wiśniewski presented a simple constant gain controller (CGC) via the Linear Quadratic Regulator (LQR) method and proposed other two attitude controllers using the periodic characteristic of the Earth magnetic field: infinite and 
finite horizon periodic controllers. In [20], Psiaki showed us how to design a class of stabilizing attitude controllers for nadir-pointing spacecraft using only magnetic actuation. Their control laws are designed using a new type of periodic linear quadratic regulator whose Riccati equation solution is approximated by a linear time-invariant solution for an averaged system. The resulting full-state feedback controller derives its periodicity from the time-varying control influence matrix which can be derived from on-board magnetometer measurements. Both authors considered the linear timevarying system taking the periodic nature of the magnetic field as an advantage to approximate the solution of the Riccati equation. A considerable amount of work has been dedicated to the problems of analysis and design of magnetic control laws in the linear case, that is, control laws for nominal operation of a satellite near its equilibrium attitude (see, e.g., $[9,17,19-25])$. Abdelrahman et al. [26] proposed an attitude control method of a satellite with only magnetic torquers as actuators via the state-dependent Riccati equation (SDRE) technique based on pseudolinear time-varying modeling of the spacecraft nonlinear dynamics. However, limited attention has been dedicated to global formulations of the problem. In $[18,27,28]$, the attitude regulation problem for Earth pointing spacecraft is studied exploiting the (quasi) periodic behavior of the system, which used the standard passivity arguments to prove local asymptotic stabilizability of open-loop equilibria. A solution to the global stabilization problem by means of full (or partial) state feedback has been studied by Lovera and Astolfi [29-32]. Conditions for almost global attitude regulation are obtained in $[29,30]$ for the case of full state feedback, and local results are derived for the case of output (attitude only) feedback. The paper [32] shows us how stability conditions similar to those given in $[29,30]$ that can be derived for control laws achieving Earth pointing for magnetically actuated spacecraft, taking also into account the effect of gravity gradient torques. For this problem, an almost global stabilization result is given for the case of full state feedback, resorting to an adaptive PD-like state feedback control law. With respect to works $[29,30,32]$ the results presented in [31] that can deal with a generic magnetically actuated satellite do not rely on restrictive assumptions on the controller parameters and guarantee that there are no trajectories of the closed-loop system along which the average controllability can be lost. These results do not rely on the (frequently adopted) periodicity assumption for the geomagnetic field along the considered orbit. The proposed framework for closed-loop stability analysis of magnetically controlled spacecraft can be also exploited to predict the effect of actuator faults on the behavior of the controlled satellite.

An alternative way to solve the active three-axis global magnetic attitude stabilization problem of a low Earth orbit satellite is suggested here. The method is based on guidance control [33], a more general control procedure. Considering an auxiliary global stabilization problem that we know an analytic solution, the method consists to use a trajectory of the globally stabilizable auxiliary system as a guide. We construct a local stabilizer that keeps the trajectory of the system in a neighborhood of a solution of the auxiliary system. In this way, the trajectory of the system tends to the equilibrium position. According to this approach and using $\mathcal{\varepsilon}$-strategies introduced by Pontryagin in the frame of differential games theory [34], the stabilizing control is constructed as a function of time defined in a small time interval and not as a feedback. From the practical point of view, $\varepsilon$-strategy is similar to stabilizer which depends on the time and position only, because it usually is implemented as a generator of piecewise constant controls. The numerical implementation of this method is based on the construction of multistep reachability sets [33]. The main contribution of this method is to solve a global stabilization problem applying local stabilization methods that avoid difficulties which we usually have in global stabilization problems, helping to overcome serious mathematical difficulties and being more effective in applications. This procedure also yields additional possibilities for the design of a stabilizer that eliminates the peak effect, that is, the large deviation of the solutions from the equilibrium position at the beginning of the stabilization process that we have when we construct stabilizers to obtain a high speed of damping of the control systems trajectories (studies of this effect are presented in [35-37]). The minimization problem used in this paper also eliminates this effect which represents a serious obstacle to guidance stabilization.

From all studies about the nonlinear controllability theory, the work [38] presents an application of this theory to the time-varying attitude dynamics of a magnetically actuated spacecraft in a Keplerian orbit in the geomagnetic field. The model considered in [38] does not include the gravitational torque and therefore the result can be applied to spherically symmetric satellites only. Considering a satellite subjected to gravity gradient torque, the controllability of the magnetic attitude of the satellite is studied here. Conditions of controllability when the satellite circular orbit is neither polar nor equatorial are obtained by local controllability theory.

It is assumed that the satellite moves along a circular orbit and the current value of the geomagnetic field with respect to the satellite axes is known due to three-axis magnetometer measurements. The position of the satellite mass center is also assumed to be known and the currents in the coils are control parameters.

\section{Guidance Stabilization Process}

Consider a control system

$$
\dot{x}(t)=f(t, x(t))+G(t, x(t)) u, \quad u \in S,
$$

where $f: R \times R^{n} \rightarrow R^{n}$ and $G: R \times R^{n} \rightarrow M(n, k)$ are sufficiently smooth functions, that is, there exist all derivatives needed in our considerations. Let $0 \in \operatorname{int} S \subset R^{k}$ be such that $f(t, \tilde{x})=0$ for all $t$, that is, $\tilde{x} \in R^{n}$ is an equilibrium position. The global stabilization problem is to find a map $u$ : $R^{n} \rightarrow S$ such that $u(\tilde{x})=0$ and the equilibrium position $\tilde{x}$ of the differential equation $\dot{x}(t)=f(t, x(t))+G(t, x(t)) u(x(t))$ is globally asymptotically stable. This problem is usually very hard to solve. One of the ways to avoid difficulties is to use a guidance control [33], a more general control procedure. Consider an auxiliary differential equation $\dot{\hat{x}}(t)=$ 
$f(t, \hat{x}(t))+w(\hat{x}(t))$ such that $\tilde{x}$ is an globally asymptotically stable equilibrium position. The trajectories of system (1), under the guidance control, are defined as solutions to the Cauchy problem:

$$
\begin{aligned}
& \dot{x}(t)=f(t, x(t))+G(t, x(t)) u(t, x, \hat{x}), \\
& \dot{\hat{x}}(t)=f(t, \hat{x}(t))+w(\hat{x}(t)), \\
& x\left(t_{0}\right)=x_{0} \\
& \hat{x}\left(t_{0}\right)=\hat{x}_{0}
\end{aligned}
$$

where the trajectory $\hat{x}(t)$, that is considered to be a "guide", tends to the equilibrium position $\tilde{x}$. Then we construct $u(t, x, \hat{x}) \in S, t \in\left[t_{0}+k \varepsilon, t_{0}+(k+1) \varepsilon\right], \varepsilon>0, k=0,1, \ldots$, such that $|\bar{x}(t)|=|x(t)-\hat{x}(t)| \rightarrow 0, t \rightarrow \infty$, where $x(\cdot)$ is a solution to (1). To this end, we linearize system (1) along the trajectory $\hat{x}(t)$ and apply the methods developed to solve the stabilization problem for linear systems. This approach works only if $\bar{x}(t)$ is sufficiently small. For this reason, we construct the control $u(t, x, \hat{x})$ solving the following minimization problem

$$
\begin{gathered}
\min _{u(t, x, \hat{x}) \in S} \max _{t \in\left[t_{0}+k \varepsilon, t_{0}+(k+1) \varepsilon\right]}|\bar{x}(t)|, \\
\dot{\bar{x}}(t)=A(t) \bar{x}(t)+B(t) u(t)-w(t), \\
t \in\left[t_{0}+k \varepsilon, t_{0}+(k+1) \varepsilon\right], \\
\bar{x}\left(t_{0}+k \varepsilon\right)=\bar{x}_{k}, \\
\bar{x}\left(t_{0}+(k+1) \varepsilon\right) \in \beta\left|\bar{x}_{k}\right| B_{n}, \quad k=0,1, \ldots,
\end{gathered}
$$

where $0<\beta<1, \varepsilon>0, A(t)=\nabla_{x} f(t, \hat{x}(t)), B(t)=G(t, \hat{x}(t))$ and $w(t)=w(\hat{x}(t))$. In this way, we generate an $\varepsilon$-strategy, that is, a sequence of controls $u$ defined on the intervals $\left[t_{0}+k \varepsilon, t_{0}+(k+1) \varepsilon\right], k=0,1$, and so on, that minimizes the norm of the solution $\bar{x}(t)$ forcing this way the solution $\bar{x}(t)$ to be small for all $t$. This implies that the trajectory $x(t)$ of (1) follows the trajectory $\hat{x}(t)$ of the auxiliary differential equation. As $\hat{x}(t) \rightarrow \tilde{x}, t \rightarrow \infty$, then $x(t) \rightarrow \tilde{x}, t \rightarrow \infty$. These controls depend on $t$ and $\bar{x}\left(t_{0}+k \varepsilon\right), k=0,1, \ldots$ This problem has a solution if the control system $\dot{\bar{x}}(t)=A(t) \bar{x}(t)+$ $B(t) u(t)-w(t), u(t) \in S$, is locally controllable.

Let $u(t) \in S, t \geq t_{0}$, be a measurable bounded control. The solution of the linearized system $\dot{\bar{x}}(t)=A(t) \bar{x}(t)+$ $B(t) u(t)-w(t)$, where $\bar{x}\left(t_{0}\right)=\bar{x}_{0}$, is denoted by $\bar{x}\left(\cdot, t_{0}\right.$, $\left.\bar{x}_{0}, u(\cdot)\right)$ and the symbol $\bar{X}\left(t_{0}, \bar{x}_{0}\right)$ is used for the set of all solutions. Define the reachability set $\overline{\mathbf{R}}\left(t_{1}, t_{0}, \bar{x}_{0}\right)=\left\{\bar{x}\left(t_{1}, t_{0}\right.\right.$, $\left.\left.\bar{x}_{0}, u(\cdot)\right) \in R^{n}: \bar{x}\left(\cdot, t_{0}, \bar{x}_{0}, u(\cdot)\right) \in \bar{X}\left(t_{0}, \bar{x}_{0}\right)\right\}$. Using the following well-known result from the control theory (see [39]), we have a possibility to check if minimization problem (3) has a solution, that is, we can prove that there exists an $\varepsilon$-strategy such that $|\bar{x}(t)|=|x(t)-\hat{x}(t)| \rightarrow 0, t \rightarrow \infty$.

Theorem 1 ([39]). The linearized system is controllable on the interval $\left[t_{0}, t_{1}\right]$, that is, $\overline{\mathbf{R}}\left(t_{1}, t_{0}, 0\right)=R^{n}$, if and only if, only the trivial solution $\bar{x}^{*}(t) \equiv 0$ to the adjoint differential equation $\dot{\bar{x}}^{*}(t)=-A^{*}(t) \bar{x}^{*}(t), t \in\left[t_{0}, t_{1}\right]$, satisfies the orthogonality condition $B^{*}(t) \bar{x}^{*}(t)=0, t \in\left[t_{0}, t_{1}\right]$. The condition
$\overline{\mathbf{R}}\left(t_{1}, t_{0}, 0\right)=R^{n}$ implies that control system (1) is controllable around the trajectory $\hat{x}(\cdot)$ on the interval $\left[t_{0}, t_{1}\right]$.

\section{Application to the Satellite Stabilization Problem}

Consider a satellite moving along a circular orbit. Introduce two Cartesian reference systems $O X_{1} X_{2} X_{3}$ and $o x_{1} x_{2} x_{3}$. The system $O X_{1} X_{2} X_{3}$ is the body reference system. The origin of this system, $O$, coincides with the satellite mass center and the axes are directed along the principal inertia axes. The system $o x_{1} x_{2} x_{3}$ is the orbital reference system. The origin $o$ coincides with the center of mass of the satellite. The axis $o x_{3}$ is directed along the radius vector of the satellite mass center and the axis $o x_{2}$ is perpendicular to the orbital plane. The angular position of the satellite with respect to the orbital system is described by three positive left hand rotations defined by the Euler angles $(\alpha, \beta, \gamma)$. Coordinates of a vector in the satellite body system, $X$, and in the orbital system, $x$, satisfy the relation $x=B X$, where $B$ is an orthogonal 3-1-2 rotation matrix with $\operatorname{det} B=1$. The components of the matrix:

$$
B=\left(\begin{array}{lll}
b_{11} & b_{12} & b_{13} \\
b_{21} & b_{22} & b_{23} \\
b_{31} & b_{32} & b_{33}
\end{array}\right)
$$

are

$$
\begin{aligned}
& b_{11}=\cos \alpha \cos \beta+\sin \alpha \sin \beta \sin \gamma, \\
& b_{12}=-\cos \alpha \sin \beta+\sin \alpha \cos \beta \sin \gamma, \\
& b_{13}=\sin \alpha \cos \gamma, \\
& b_{21}=\sin \beta \cos \gamma, \\
& b_{22}=\cos \beta \cos \gamma, \\
& b_{23}=-\sin \gamma, \\
& b_{31}=-\sin \alpha \cos \beta+\cos \alpha \sin \beta \sin \gamma, \\
& b_{32}=\sin \alpha \sin \beta+\cos \alpha \cos \beta \sin \gamma, \\
& b_{33}=\cos \alpha \cos \gamma .
\end{aligned}
$$

Denote by $b_{i}, i=1,2,3$, the rows of the matrix $B$. Obviously $b_{3}=b_{1} \times b_{2}$.

The satellite is equipped with three magnetic coils oriented along three orthogonal axes. Let $\Omega$ be the vector of angular velocity in the body axes. The rotation of the satellite subjected to gravity gradient torque is described by the following equations:

$$
\begin{gathered}
J \dot{\Omega}=-\Omega \times J \Omega+3 \omega^{2} b_{3} \times J b_{3}+U \times F, \\
\dot{b}_{i}=b_{i} \times\left(\Omega-\omega b_{2}\right), \quad i=1,2,3,
\end{gathered}
$$

where

(i) the tensor of inertia $J$ is a diagonal matrix with the diagonal elements $J_{1}, J_{2}, J_{3}$, such that $J_{3}<J_{1} \leq J_{2}$, 
(ii) the vector $F$ of the geomagnetic field in the body axes is given by $F=B^{*} f$, where $f=\mu_{0} \mu_{m} r^{-3}(\cos \nu \sin \tau$, $\cos \tau,-2 \sin \nu \sin \tau)$,

(iii) $\tau$ is the orbital inclination, that is, the angle between the equatorial and orbital planes,

(iv) $v=\omega t+\varphi_{0}$ is the argument of latitude,

(v) $\omega=\left(\mu_{g} / r^{3}\right)^{1 / 2} \approx 0.001 \mathrm{~s}^{-1}$ is the angular velocity of the orbital motion,

(vi) $r=7.4 \times 10^{6} \mathrm{~m}$ is the radius of the orbit,

(vii) $\mu_{m}=8.06 \times 10^{22} \mathrm{~A} \cdot \mathrm{m}^{2}$ is the Earth magnetic dipole moment,

(viii) $\mu_{g}=3.986 \times 10^{14} \mathrm{~m}^{3} / \mathrm{c}^{2}$ is the Earth gravitational parameter,

(ix) $\mu_{0}=4 \pi \times 10^{-7} \mathrm{H} / \mathrm{m}$ is the magnetic parameter,

(x) $\varphi_{0}$ describe the initial position of the mass center in the orbit,

(xi) the vector $U$ has the components $U_{i}=I_{i} \rho L, i=1,2$, 3 , where $I_{i}$ stands for the current in the ith coil, $\rho$ is the number of turns, and $L$ is the area of a loop.

The currents $I_{i}, i=1,2,3$, are control parameters. At the initial moment $t_{0}$, the satellite has an angular position that corresponds to the matrix $B\left(t_{0}\right)=B_{0}$ and the angular velocity $\Omega\left(t_{0}\right)=\Omega_{0}$. The problem is to find control laws $I_{i}=$ $I_{i}(F)$ that drive $B(t)$ and angular velocity to $I$ and $(0, \omega, 0)$, respectively. Considering the Lyapunov function, known as the Jacobi integral,

$$
\begin{aligned}
V(\alpha, \beta, \gamma, \Omega)= & \frac{1}{2}\left(\Omega-\omega b_{2}\right)^{*} J\left(\Omega-\omega b_{2}\right)+\frac{3}{2} \omega^{2} b_{3}^{*} J b_{3} \\
& -\frac{1}{2} \omega^{2} b_{2}^{*} J b_{2}-\frac{1}{2} \omega^{2}\left(3 J_{3}-J_{2}\right),
\end{aligned}
$$

we see that this is a stable equilibrium position $[18,28]$.

Consider the auxiliary system:

$$
\begin{gathered}
J \dot{\hat{\Omega}}=-\hat{\Omega} \times J \hat{\Omega}+3 \omega^{2} \hat{b}_{3} \times J \hat{b}_{3}+W, \\
\dot{\hat{b}}_{i}=\hat{b}_{i} \times\left(\hat{\Omega}-\omega \hat{b}_{2}\right), \quad i=1,2,3,
\end{gathered}
$$

where

$$
\begin{aligned}
W= & \sum_{i=1}^{3} k_{i} r_{i} \times \hat{b}_{i}-\theta\left(\hat{\Omega}-\omega \hat{b}_{2}\right) \\
& +\omega\left(J\left(\hat{b}_{2} \times \hat{\Omega}\right)+\hat{\Omega} \times J \hat{b}_{2}+\hat{b}_{2} \times J \hat{\Omega}\right) \\
& -\omega^{2}\left(\hat{b}_{2} \times J \hat{b}_{2}+3 \hat{b}_{3} \times J \hat{b}_{3}\right)
\end{aligned}
$$

for $k_{i}>0, k_{i} \neq k_{j}, i, j=1,2,3, i \neq j,\left(\begin{array}{lll}r_{1} & r_{2} & r_{3}\end{array}\right)=I, \theta>0$, $\hat{\Omega} \in R^{3}$ and $\widehat{B}=\left(\begin{array}{lll}\hat{b}_{1} & \hat{b}_{2} & \hat{b}_{3}\end{array}\right)^{*}$ has the same properties as $B$.

Theorem 2. System (8)-(9) possesses an asymptotically stable equilibrium position $\widetilde{\Omega}=(0, \omega, 0), \widetilde{b}_{i}=r_{i}, i=1,2,3$.
Using the following lemma, the proof of Theorem 2 is trivial.

Lemma 3. The system

$$
\begin{gathered}
J \Theta=-\Theta \times J \Theta+P, \\
\dot{\hat{b}}_{i}=\hat{b}_{i} \times \Theta, \quad i=1,2,3,
\end{gathered}
$$

where $P=\sum_{i=1}^{3} k_{i} r_{i} \times \hat{b}_{i}-\theta \Theta$, has the asymptotically stable equilibrium position $\Theta=(0,0,0), \hat{b}_{i}=r_{i}, i=1,2,3$.

Proof. Considering the Lyapunov function $V=(\langle\Theta, J \Theta\rangle+$ $\left.\sum_{i=1}^{3} k_{i}\left(\hat{b}_{i}-r_{i}\right)^{2}\right) / 2$ and following the proof of theorem in [40], we get the result.

Remark: We can also see the proof of this lemma in [41].

Proof of Theorem 2. Using the equality:

$$
\widehat{\Omega}=\Theta+\omega \hat{b}_{2}
$$

in (8), we have

$$
\begin{gathered}
J \dot{\Theta}=-\Theta \times J \Theta+G, \\
\dot{\hat{b}}_{i}=\hat{b}_{i} \times \Theta, \quad i=1,2,3,
\end{gathered}
$$

where

$$
\begin{aligned}
G= & W-\omega\left(J\left(\hat{b}_{2} \times \Theta\right)+\Theta \times J \hat{b}_{2}+\hat{b}_{2} \times J \Theta\right) \\
& -\omega^{2}\left(\hat{b}_{2} \times J \hat{b}_{2}-3 \hat{b}_{3} \times J \hat{b}_{3}\right) .
\end{aligned}
$$

Considering

$$
\begin{aligned}
W= & \sum_{i=1}^{3} k_{i} r_{i} \times \hat{b}_{i}-\theta \Theta+\omega\left(J\left(\hat{b}_{2} \times \Theta\right)+\Theta \times J \hat{b}_{2}+\hat{b}_{2} \times J \Theta\right) \\
& +\omega^{2}\left(\hat{b}_{2} \times J \hat{b}_{2}-3 \hat{b}_{3} \times J \hat{b}_{3}\right)
\end{aligned}
$$

and using Lemma 3, we see that system (12)-(13) has the following asymptotically stable equilibrium position

$$
\Theta=(0,0,0), \quad \hat{b}_{i}=r_{i}, \quad i=1,2,3 .
$$

Using again equality (11) in (14)-(15), we obtain the result.

Auxiliary system (8)-(9) will be a guide for control system (6). Using the techniques presented in previous section, we numerically create a control $U$ such that system (6) follows auxiliary system (8)-(9). Finding $U$, we get the currents $I_{i}, i=1,2,3$. Its only necessity to prove that the linear system of minimization problem (3) is locally controllable. Considering $\lambda=(\alpha, \beta, \gamma)$, the corresponding adjoint system takes the form:

$$
\begin{aligned}
& \dot{\bar{\Omega}}^{*}=A_{1} \bar{\Omega}^{*}+A_{2} \bar{\lambda}^{*}, \\
& \dot{\bar{\lambda}}^{*}=A_{3} \bar{\Omega}^{*}+A_{4} \bar{\lambda}^{*},
\end{aligned}
$$


where $\bar{\lambda}^{*}, \bar{\Omega}^{*} \in R^{3}, A_{1}=\left(\nabla_{\Omega} \Phi\right)^{*}, A_{2}=\Upsilon^{*}, A_{3}=\left(\nabla_{\lambda} \Psi\right)^{*}$, $A_{4}=\left(\nabla_{\lambda} \Upsilon \cdot \hat{\Omega}\right)^{*}$,

$$
\begin{aligned}
& \Phi=\left(\begin{array}{c}
\frac{J_{3}-J_{2}}{J_{1}} \hat{\Omega}_{2} \hat{\Omega}_{3} \\
\frac{J_{1}-J_{3}}{J_{2}} \hat{\Omega}_{1} \hat{\Omega}_{3} \\
\frac{J_{2}-J_{1}}{J_{3}} \hat{\Omega}_{1} \hat{\Omega}_{2}
\end{array}\right), \\
& \Upsilon=-\left(\begin{array}{ccc}
\frac{\sin \hat{\beta}}{\cos \hat{\gamma}} & \frac{\cos \hat{\beta}}{\cos \hat{\gamma}} & 0 \\
\sin \hat{\beta} \tan \hat{\gamma} & \cos \hat{\beta} \tan \hat{\gamma} & 1 \\
\cos \hat{\beta} & -\sin \hat{\beta} & 0
\end{array}\right), \\
& \Psi=-3 \omega^{2}\left(\begin{array}{c}
\frac{J_{3}-J_{2}}{J_{1}} \cos \hat{\alpha} \cos \hat{\gamma} \\
\times(\sin \hat{\alpha} \sin \hat{\beta}+\cos \hat{\alpha} \cos \hat{\beta} \sin \hat{\gamma}) \\
\frac{J_{1}-J_{3}}{J_{2}} \cos \hat{\alpha} \cos \hat{\gamma} \\
\times(\cos \hat{\alpha} \sin \hat{\beta} \sin \hat{\gamma}-\sin \hat{\alpha} \cos \hat{\beta}) \\
\frac{J_{2}-J_{1}}{J_{3}}(\cos \hat{\alpha} \sin \hat{\beta} \sin \hat{\gamma}-\sin \hat{\alpha} \cos \hat{\beta}) \\
\times(\sin \hat{\alpha} \sin \hat{\beta}+\cos \hat{\alpha} \cos \hat{\beta} \sin \hat{\gamma})
\end{array}\right) .
\end{aligned}
$$

If $\bar{\Omega}^{*} \equiv 0$, then the first equation of the adjoint system implies $\bar{\lambda}^{*} \equiv 0$, because $\operatorname{det} A_{2} \neq 0$. The orthogonality condition from Theorem 1 can be written in two equivalent forms $\bar{\Omega}^{*} \times F=0$ and $\bar{\Omega}^{*}=\xi F$. Differentiating the latter, we obtain $\dot{\bar{\Omega}}^{*}=\dot{\xi} F+\xi \dot{F}$. Substituting this for $\dot{\bar{\Omega}}^{*}$ in the first equation of the adjoint system, we have $\bar{\lambda}^{*}=A_{2}^{-1}\left(\dot{\xi} F+\xi\left(\dot{F}-A_{1} F\right)\right)$. Differentiating the first equation of the adjoint system and substituting the expression for $\bar{\lambda}^{*}$ obtained above, we get $\ddot{\bar{\Omega}}^{*}=$ $\left(\dot{A}_{2}+A_{2} A_{4}\right) A_{2}^{-1}\left(\dot{\xi} F+\xi\left(\dot{F}-A_{1} F\right)\right)+\xi\left(A_{2} A_{3} F+\dot{A}_{1} F+A_{1} \dot{F}\right)+$ $\dot{\xi} A_{1} F$. Differentiating $\bar{\Omega}^{*} \times F=0$ twice, we get $\ddot{\bar{\Omega}}^{*} \times F+$ $2 \dot{\bar{\Omega}}^{*} \times \dot{F}+\bar{\Omega}^{*} \times \ddot{F}=0$. Substituting the above formulas for $\bar{\Omega}^{*}$ and its derivatives, we obtain $\dot{\xi} v_{1}+\xi v_{2}=0$, where

$$
\begin{aligned}
v_{1}= & \left(\left(\dot{A}_{2}+A_{2} A_{4}\right) A_{2}^{-1} F+A_{1} F\right) \times F+2 F \times \dot{F}, \\
v_{2}= & \left(\left(\dot{A}_{2}+A_{2} A_{4}\right) A_{2}^{-1}\left(\dot{F}-A_{1} F\right)+\left(A_{2} A_{3}+\dot{A_{1}}\right) F+A_{1} \dot{F}\right) \\
& \times F+F \times \ddot{F}
\end{aligned}
$$

are real analytic functions. From Theorem 1 we see that to prove the local controllability of the linearized system of (6) it suffices to show that $\bar{\Omega}^{*} \equiv 0$ on any interval of time. This happens, for example, if the vectors $v_{1}$ and $v_{2}$ are linearly independent. Thus we have the following result.

Lemma 4. If $v_{1} \times v_{2} \neq 0$ for almost all $v \in[0,2 \pi]$, then the linearized system of (6) is controllable.
Assume that the satellite circular orbit is neither polar nor equatorial. In this case, with the help of the symbolic computation system Mathematica, we prove that the conditions of Lemma 4 are satisfied for $J_{1}=J_{2}=J_{3}$ and $\widehat{\Omega}$ is sufficiently small and for $J_{3}<J_{1} \leq J_{2}$ and $(\hat{\Omega}, \hat{\lambda})$ in a neighborhood of the stable equilibrium position. See the two following examples to each one of these cases.

Example 5. Consider $J_{1}=J_{2}=J_{3}=1, \hat{\alpha}=\hat{\beta}=\hat{\gamma}=0$ and $\widehat{\Omega} \equiv 0$. Using the symbolic computation system Mathematica we obtain

$$
\begin{aligned}
v_{1}= & \left(v_{1}^{(1)}, v_{1}^{(2)}, v_{1}^{(3)}\right) \\
= & \left(3 \omega^{2} \sin 2 \tau \sin \nu, 3 \omega^{2} \sin 2 \nu \sin ^{2} \tau, 3 \omega^{2} \sin 2 \tau \cos \nu\right), \\
v_{2}= & \left(v_{2}^{(1)}, v_{2}^{(2)}, v_{2}^{(3)}\right) \\
= & \left(-3 \omega \sin 2 \tau \cos \nu, 6 \omega \sin ^{2} \tau\left(\cos ^{2} \nu+2 \sin ^{2} \nu\right),\right. \\
& 3 \omega \sin 2 \tau \sin \nu) .
\end{aligned}
$$

As

$$
\begin{aligned}
\operatorname{det}\left(\begin{array}{cc}
v_{1}^{(1)} & v_{2}^{(1)} \\
v_{1}^{(3)} & v_{2}^{(3)}
\end{array}\right) & =9 \omega^{3} \sin ^{2} 2 \tau \cdot \operatorname{det}\left(\begin{array}{cc}
\sin \nu & -\cos \nu \\
\cos \nu & \sin \nu
\end{array}\right) \\
& =9 \omega^{3} \sin ^{2} 2 \tau \neq 0,
\end{aligned}
$$

for all $\tau \in] 0, \pi / 2$ [, then the vectors $v_{1}$ and $v_{2}$ are linearly independent. This proves the conditions of Lemma 4.

Example 6. Now, consider any $J_{3}<J_{1}<J_{2}, \hat{\alpha}=\hat{\beta}=\hat{\gamma}=0$ and $\hat{\Omega}=(0, \omega, 0)$. Using the symbolic computation system Mathematica we obtain $v_{1} \times v_{2}=(a, b, c)$, where

$$
\begin{gathered}
a=\left(\begin{array}{l}
\frac{a_{1} \cos \tau \sin ^{3} \tau}{J_{1}^{2} J_{2} J_{3}^{2}}\left(\frac{3}{4}\left(5 J_{1}-J_{2}+J_{3}\right) J_{3}+J_{1}\left(J_{1}-J_{2}+2 J_{3}\right)\right) \\
-\frac{a_{2} \sin ^{2} \tau \sin 2 \tau}{4 J_{1} J_{3}^{2}} \\
\frac{a_{1} \cos \tau \sin ^{3} \tau}{J_{1}^{2} J_{2} J_{3}^{2}}\left(\frac{1}{4}\left(5 J_{1}-J_{2}+J_{3}\right) J_{3}-J_{1}\left(J_{1}-J_{2}+2 J_{3}\right)\right) \\
+\frac{a_{2} \sin ^{2} \tau \sin 2 \tau}{4 J_{1} J_{3}^{2}} \\
b= \\
\quad\left(\begin{array}{c}
\cos \nu \\
\cos 3 \nu
\end{array}\right), \\
\frac{b_{1} \cos { }^{2} \tau \sin ^{2} \tau}{J_{1}^{2} J_{2} J_{3}}-\frac{b_{2} \sin ^{2} 2 \tau}{2 J_{1} J_{2} J_{3}^{2}} \\
\frac{b_{1} \cos ^{2} \tau \sin ^{2} \tau}{J_{1}^{2} J_{2} J_{3}}+\frac{b_{2} \sin ^{2} 2 \tau}{2 J_{1} J_{2} J_{3}^{2}}
\end{array}\right)^{*} \cdot\left(\begin{array}{c}
1 \\
\cos 2 \nu
\end{array}\right),
\end{gathered}
$$




$$
\begin{aligned}
& c=\left(\begin{array}{l}
\frac{c_{1} \cos \tau \sin ^{3} \tau}{J_{1}^{2} J_{2} J_{3}^{2}}\left(\frac{1}{4}\left(5 J_{1}-J_{2}+J_{3}\right) J_{3}+3 J_{1}\left(J_{1}-J_{2}+2 J_{3}\right)\right) \\
-\frac{c_{2} \cos \tau \sin ^{3} \tau}{4 J_{1}^{2} J_{3}} \\
\frac{c_{1} \cos \tau \sin ^{3} \tau}{J_{1}^{2} J_{2} J_{3}^{2}}\left(\frac{1}{4}\left(5 J_{1}-J_{2}+J_{3}\right) J_{3}-J_{1}\left(J_{1}-J_{2}+2 J_{3}\right)\right) \\
-\frac{c_{2} \cos \tau \sin ^{3} \tau}{4 J_{1}^{2} J_{3}}
\end{array}\right)^{*} \\
& \cdot\left(\begin{array}{c}
\sin v \\
\sin 3 v
\end{array}\right) \text {, }
\end{aligned}
$$

with

$$
\begin{aligned}
& a_{1}=-\left(J_{1}^{2}\left(2 J_{2}+3 J_{3}\right)+4 J_{2}\left(-J_{2}+J_{3}\right) J_{3}\right. \\
& \left.+J_{1}\left(-2 J_{2}^{2}+3 J_{2} J_{3}-3 J_{3}^{2}\right)\right) \omega^{3}, \\
& a_{2}=-\left(J_{1}-J_{2}+2 J_{3}\right)\left(2 J_{1}^{2}+7 J_{3}\left(-J_{2}+J_{3}\right)\right. \\
& \left.+J_{1}\left(-2 J_{2}+3 J_{3}\right)\right) \omega^{3}, \\
& b_{1}=\frac{1}{2}\left(-5 J_{1}+J_{2}-J_{3}\right)\left(J_{1}^{2}\left(2 J_{2}+3 J_{3}\right)+4 J_{2}\left(-J_{2}+J_{3}\right) J_{3}\right. \\
& \left.+J_{1}\left(-2 J_{2}^{2}+3 J_{2} J_{3}-3 J_{3}^{2}\right)\right) \omega^{3}, \\
& b_{2}=\frac{1}{2}\left(J_{1}-J_{2}+2 J_{3}\right)\left(2 J_{1}^{2}\left(J_{2}+3 J_{3}\right)+J_{2}\left(-J_{2}+J_{3}\right) J_{3}\right. \\
& \left.+J_{1}\left(-2 J_{2}^{2}+3 J_{2} J_{3}-6 J_{3}^{2}\right)\right) \omega^{3}, \\
& c_{1}=\left(J_{2}\left(-J_{2}+J_{3}\right) J_{3}+2 J_{1}^{2}\left(J_{2}+3 J_{3}\right)\right. \\
& \left.+J_{1}\left(-2 J_{2}^{2}+3 J_{2} J_{3}-6 J_{3}^{2}\right)\right) \omega^{3}, \\
& c_{2}=\left(-5 J_{1}+J_{2}-J_{3}\right)\left(2 J_{1}^{2}+7 J_{3}\left(-J_{2}+J_{3}\right)\right. \\
& \left.+J_{1}\left(-2 J_{2}+3 J_{3}\right)\right) \omega^{3} \text {. }
\end{aligned}
$$

Suppose that $b \equiv 0$. As

$$
\operatorname{det}\left(\begin{array}{cc}
\frac{\cos ^{2} \tau \sin ^{2} \tau}{J_{1}^{2} J_{2} J_{3}} & -\frac{\sin ^{2} 2 \tau}{2 J_{1} J_{2} J_{3}^{2}} \\
\frac{\cos ^{2} \tau \sin ^{2} \tau}{J_{1}^{2} J_{2} J_{3}} & \frac{\sin ^{2} 2 \tau}{2 J_{1} J_{2} J_{3}^{2}}
\end{array}\right)=\frac{\cos ^{2} \tau \sin ^{2} \tau \sin ^{2} 2 \tau}{J_{1}^{3} J_{2}^{2} J_{3}^{3}} \neq 0,
$$

for all $\tau \in] 0, \pi / 2\left[\right.$, then we have $b_{1}=b_{2}=0$. From triangular inequality for moments of inertia, we have $J_{2}<J_{1}+J_{3}$. Thus $b_{1}=0$ is equivalent to

$$
2 J_{1} J_{2}\left(J_{1}-J_{2}\right)=-3 J_{1} J_{3}\left(J_{1}-J_{3}\right)-3 J_{1} J_{2} J_{3}+4 J_{2} J_{3}\left(J_{2}-J_{3}\right)
$$

and $b_{2}=0$ is equivalent to

$$
6 J_{1} J_{3}\left(J_{1}-J_{3}\right)+J_{2} J_{3}\left(J_{3}-J_{2}\right)+3 J_{1} J_{2} J_{3}=-2 J_{1} J_{2}\left(J_{1}-J_{2}\right) .
$$

From (24) and (25) we get

$$
3 J_{1} J_{3}\left(J_{1}-J_{3}\right)=-3 J_{2} J_{3}\left(J_{2}-J_{3}\right) \text {. }
$$

Dividing both sides of (26) by $J_{1} J_{2} J_{3}$, we have

$$
3\left(\frac{J_{1}-J_{3}}{J_{2}}\right)=-3\left(\frac{J_{2}-J_{3}}{J_{1}}\right),
$$

that is a contradiction. Thus we have $b \equiv 0$ to a finite number of points $\nu$. This proves the conditions of Lemma 4 .

\section{Stabilization Algorithm and Numerical Simulation}

Consider the following stabilization algorithm based on the previous results and a numerical simulation.

4.1. Stabilization Algorithm. The stabilization algorithm presented (see Algorithm 1) is a simple illustration of the previous method that we can implement to generate a sequence of controls $U$ defined on the intervals $\left[t_{0}+k \varepsilon, t_{0}+(k+1) \varepsilon\right]$, $k=0,1$, and so on. This sequence of controls can be used in a real situation in real time.

4.2. Numerical Simulation. To test the algorithm we consider satellite (6) and auxiliary system (8)-(9) having the same initial position in $t_{0}=0: \Omega(0)=\widehat{\Omega}(0)=(0,0,0)$ and $B(0)=$ $\hat{B}(0)$, where $(\alpha, \beta, \gamma)=(\pi / 3, \pi / 12,-\pi / 9)$ and consider the following parameters: the orbital inclination $\tau=\pi / 3$, the tensor of inertia $J=\operatorname{diag}(1.7,1.8,1.4)$, the initial position of the mass center in the orbit $\varphi_{0}=-\pi / 2, \theta=1, k_{1}=0.1$, $k_{2}=0.2, k_{3}=0.3, \varepsilon=6, n=10$ and $m=3$.

Implementing an $\varepsilon$-strategy in the form of three-step reachability set construction algorithm, that is, considering $m=3$ in the above algorithm, we obtain the results of the numerical simulation that are shown in Figures 1 and 2. This $\mathcal{E}$-strategy consists in the following. First, we divide the intervals of time $\left[t_{0}+k \varepsilon, t_{0}+(k+1) \varepsilon\right], k=0,1$, and so on, on three subsets with the same size $\left[t_{0}+(k+(j-1) / 3) \varepsilon, t_{0}+(k+\right.$ $j / 3) \varepsilon], j=1,2,3$, and using the Euler's formula, we obtain approximations to $(\bar{\Omega}, \bar{B})_{3 k+j}=(\bar{\Omega}, \bar{B})\left(t_{0}+(k+j / 3) \varepsilon\right)$ which depend of piecewise constant controls $U_{3 k+j} \in\left[t_{0}+(k+\right.$ $\left.(j-1) / 3) \varepsilon, t_{0}+(k+j / 3) \varepsilon\right]$. Finally, using an numerical algorithm to calculate the minimum of $\mid\left((\bar{\Omega}, \bar{B})_{3 k+1}\right.$, $\left.(\bar{\Omega}, \bar{B})_{3 k+2},(\bar{\Omega}, \bar{B})_{3 k+3}\right) \mid$, we obtain these piecewise constant controls. Then we have $U$ and therefore we find the control laws $I_{i}, i=1,2,3$, that drive system (6) to the equilibrium position. Figure 1 represents the norm of the difference between the trajectory of satellite (6) and the trajectory of auxiliary system (8)-(9), and Figure 2 represents the norm of the difference between the trajectory of satellite (6) and the equilibrium position.

\section{Conclusions}

The problem of active three-axis magnetic attitude stabilization of a low Earth orbit satellite was studied. In this paper it was proposed an alternative approach to the existing 


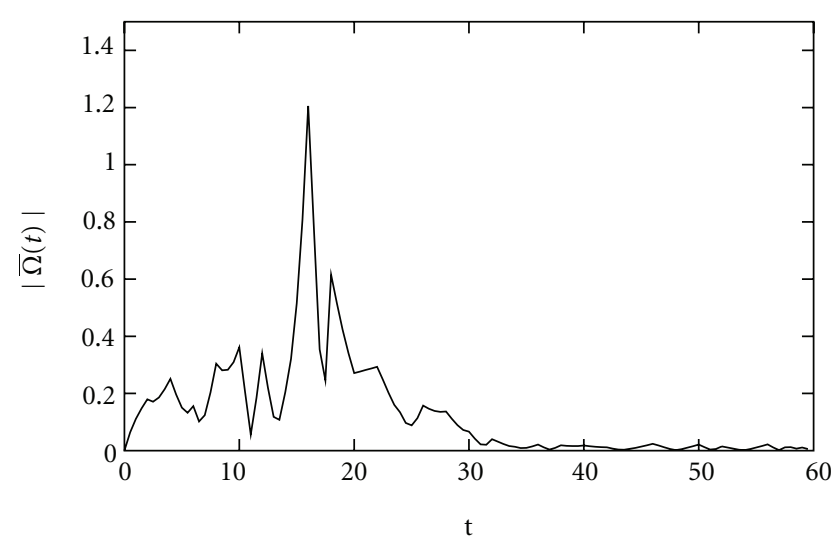

(a)

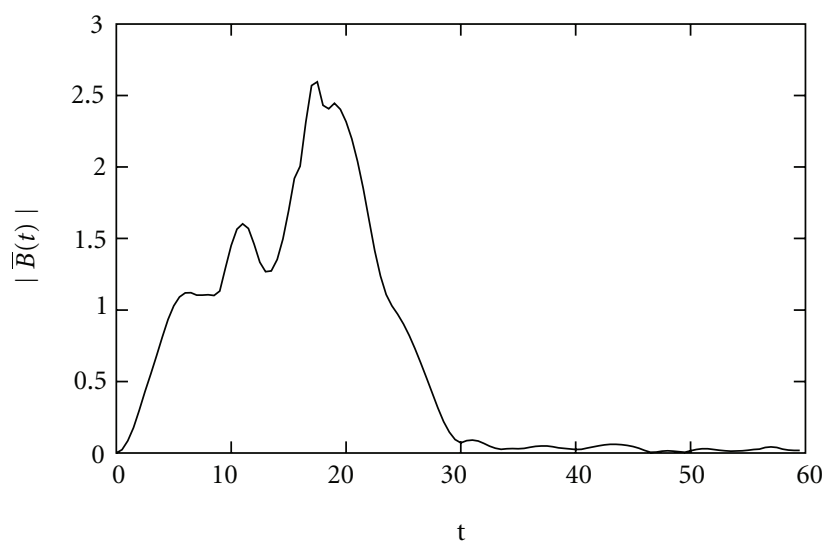

(b)

Figure 1: Norm of the difference between the satellite trajectory and the guide along the time (sec).

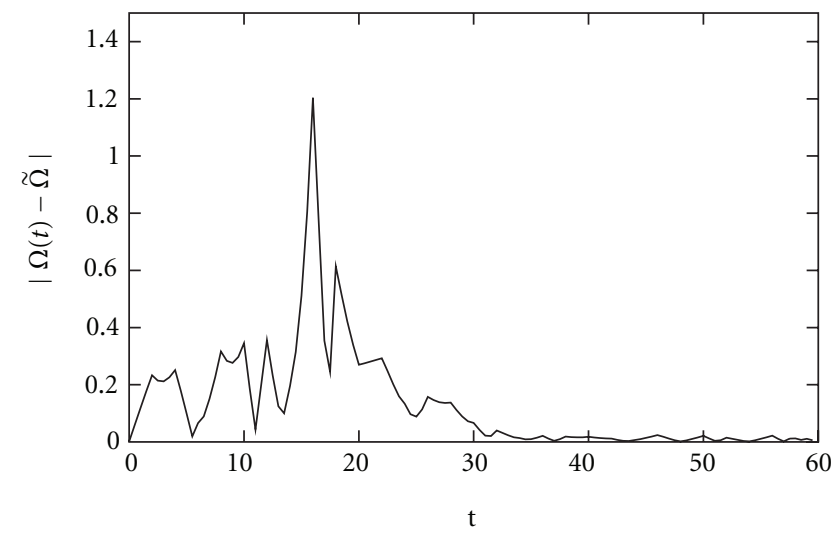

(a)

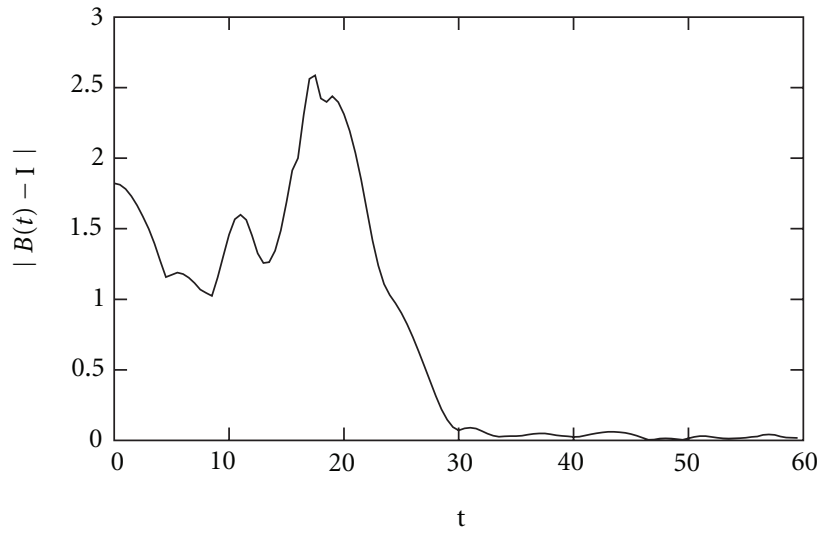

(b)

FIGURE 2: Norm of the difference between the satellite trajectory and the equilibrium position along the time (sec).

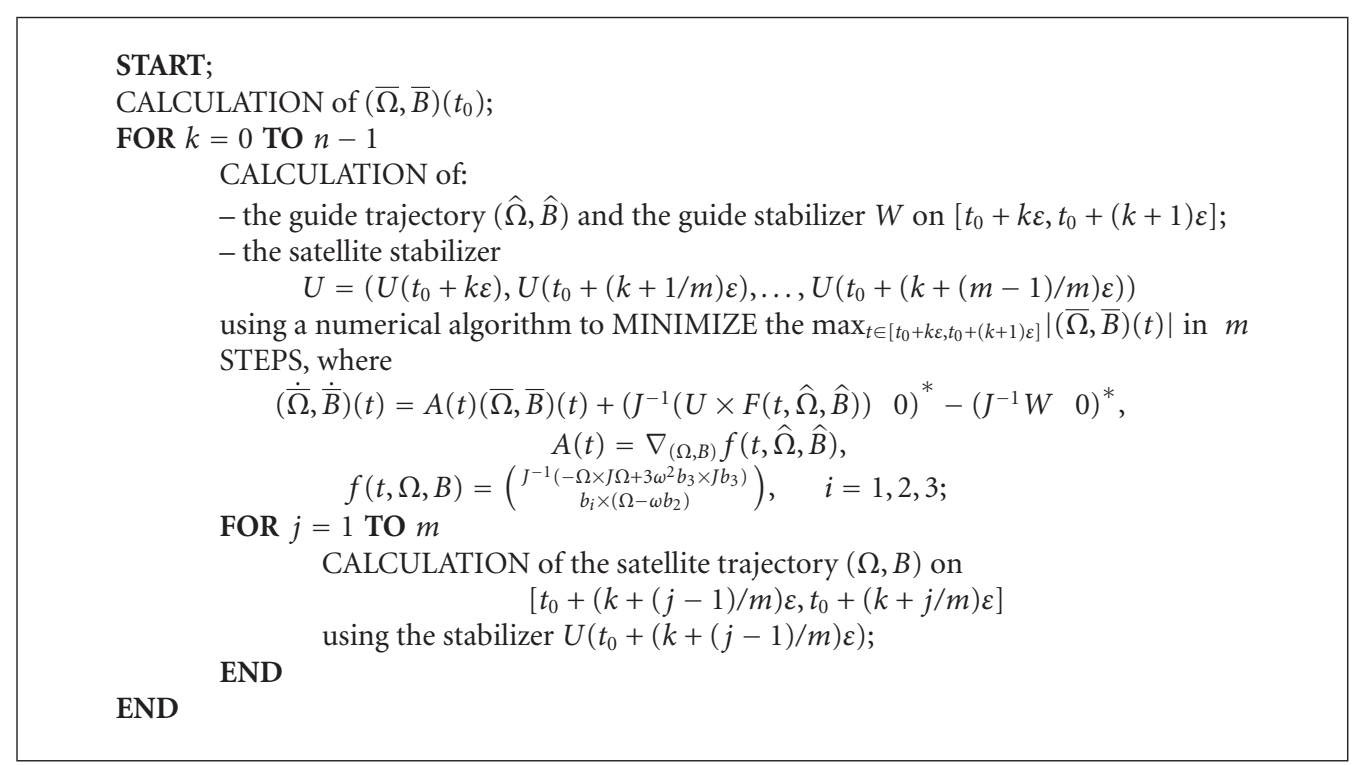


methods. The method used in the stabilization of satellite is based on guidance control, a more general control procedure. Conditions of controllability were also obtained in this study. According to these conditions and using $\mathcal{E}$-strategies, the stabilizing control was constructed as a function of time defined in a small time interval and not as a feedback. The simulation results obtained using the algorithm proposed prove the feasibility of the approach presented here. This simple approach using local stabilization methods solves the complex and difficult global stabilization problem.

\section{Nomenclature}

Throughout this paper we will use the following notations: $M(n, m)$ : The set of $n$-by- $m$ real matrices

$R: \quad$ The set of real numbers

$R^{n}: \quad$ The usual $n$-dimensional space of vectors

$x=\left(x_{1}, \ldots, x_{n}\right)$, where $x_{i} \in R, i=\overline{1, n}$

$\nabla_{x} f: \quad$ The Jacobian matrix of the function $f$

$\langle x, y\rangle: \quad$ The inner product of two vectors $x$ and $y$ in $R^{n}$

$x \times y$ : The vector product of two 3-dimensional vectors $x$ and $y$

$|x|: \quad$ The Euclidean norm of a vector $x \in R^{n}$

$|A|: \quad$ The matrix $\infty$-Norm of a real matrix $A=\left(a_{i j}\right)$, $i=\overline{1, m}, j=\overline{1, n}$

$B_{n}: \quad$ The unit ball in $R^{n}: B_{n}=\left\{x \in R^{n}:|x| \leq 1\right\}$

I: $\quad$ The identity matrix

$A^{*}$ : $\quad$ The transposed matrix of a real matrix $A$

$A^{-1}$ : $\quad$ The inverse matrix of a real matrix $A$

int $H$ : The interior of a set $H \subset R^{n}$.

\section{Acknowledgments}

This work was supported by the Portuguese Foundation for Science and Technology (FCT), the Portuguese Operational Programme for Competitiveness Factors (COMPETE), the Portuguese Strategic Reference Framework (QREN), and the European Regional Development Fund (FEDER).

\section{References}

[1] J. T.-Y. Wen and K. Kreutz-Delgado, "The attitude control problem," IEEE Transactions on Automatic Control, vol. 36, no. 10, pp. 1148-1162, 1991.

[2] O.-E. Fjellstad and T. I. Fossen, "Comments on "The attitude control problem"', IEEE Transactions on Automatic Control, vol. 39, no. 3, pp. 699-700, 1994.

[3] F. Caccavale and L. Villani, "Output feedback control for attitude tracking," Systems and Control Letters, vol. 38, no. 2, pp. 91-98, 1999.

[4] M. R. Akella, "Rigid body attitude tracking without angular velocity feedback," Systems and Control Letters, vol. 42, no. 4, pp. 321-326, 2001.

[5] C. I. Byrnes and A. Isidori, "On the attitude stabilization of rigid spacecraft," Automatica, vol. 27, no. 1, pp. 87-95, 1991.

[6] P. Morin, C. Samson, J.-B. Pomet, and Z.-P. Jiang, "Time-varying feedback stabilization of the attitude of a rigid spacecraft with two controls," Systems and Control Letters, vol. 25, no. 5, pp. 375-385, 1995.
[7] H. Iida and K. Ninomiya, "A new approach to magnetic angular momentum management for large scientific satellites," NEC Research and Development, vol. 37, no. 1, pp. 60-77, 1996.

[8] L. D. D. Ferreira and J. J. Cruz, "Attitude and spin rate control of a spinning satellite using geomagnetic field," Journal of Guidance, Control, and Dynamics, vol. 14, no. 1, pp. 216-218, 1991.

[9] M. E. Pittelkau, "Optimal periodic control for spacecraft pointing and attitude determination," Journal of Guidance, Control, and Dynamics, vol. 16, no. 6, pp. 1078-1084, 1993.

[10] K. T. Alfriend, "Magnetic attitude control system for dual-spin satellites," AIAA Journal, vol. 13, no. 6, pp. 817-822, 1975.

[11] J. S. White, F. H. Shigemoto, and K. Bourquin, "Satellite attitude control utilizing the Earth's magnetic field," Tech. Rep. D-1068, NASA, Washington, DC, USA, 1961.

[12] A. C. Stickler and K. T. Alfriend, "Elementary magnetic attitude control system," Journal of Spacecraft and Rockets, vol. 13, no. 5, pp. 282-287, 1976.

[13] F. Martel, P. K. Pal, and M. L. Psiaki, "Active magnetic control system for gravity gradient stabilized spacecraft," in Proceedings of the 2nd Annual AIAA/USU Conference on Small Satellites, Logan, Utah, USA, September 1988.

[14] K. L. Musser and W. L. Ebert, "Autonomous spacecraft attitude control using magnetic torquing only," in Proceedings of the Flight Mechanics/Estimation Theory Symposium, pp. 23-38, NASA, May 1989.

[15] R. Wiśniewski, "Nonlinear control for satellite detumbling based on magnetic torquing," in Proceedings of the 22nd (JSDE) Joint Service Data Exchange for Guidance, Navigation and Control, Scottsdale, Ariz, USA, October-November 1994.

[16] R. Wiśniewski and M. Blanke, "Three-axis satellite attitude control based on magnetic torquing," in Proceedings of the 13th IFAC World Congress, San Francisco, Calif, USA, June 1996.

[17] E. Silani and M. Lovera, "Magnetic spacecraft attitude control: a survey and some new results," Control Engineering Practice, vol. 13, no. 3, pp. 357-371, 2005.

[18] R. Wiśniewski and M. Blanke, "Fully magnetic attitude control for spacecraft subject to gravity gradient," Automatica, vol. 35, no. 7, pp. 1201-1214, 1999.

[19] R. Wiśniewski, "Linear time-varying approach to satellite attitude control using only electromagnetic actuation," Journal of Guidance, Control, and Dynamics, vol. 23, no. 4, pp. 640$647,2000$.

[20] M. L. Psiaki, "Magnetic torquer attitude control via asymptotic periodic linear quadratic regulation," Journal of Guidance, Control, and Dynamics, vol. 24, no. 2, pp. 386-394, 2001.

[21] R. Wiśniewski and F. L. Markley, "Optimal magnetic attitude control," in Proceedings of the 14th IFAC World Congress, Beijing, China, July 1999.

[22] M. Lovera, E. De Marchi, and S. Bittanti, "Periodic attitude control techniques for small satellites with magnetic actuators," IEEE Transactions on Control Systems Technology, vol. 10, no. 1, pp. 90-95, 2002.

[23] H. Yan, I. M. Ross, and K. T. Alfriend, "Pseudospectral feedback control for three-axis magnetic attitude stabilization in elliptic orbits," Journal of Guidance, Control, and Dynamics, vol. 30, no. 4, pp. 1107-1115, 2007.

[24] M. Corno and M. Lovera, "Spacecraft attitude dynamics and control in the presence of large magnetic residuals," Control Engineering Practice, vol. 17, no. 4, pp. 456-468, 2009.

[25] T. Pulecchi, M. Lovera, and A. Varga, "Optimal discrete-time design of three-axis magnetic attitude control laws," IEEE Transactions on Control Systems Technology, vol. 18, no. 3, pp. 714-722, 2010. 
[26] M. Abdelrahman, I. Chang, and S.-Y. Park, "Magnetic torque attitude control of a satellite using the state-dependent Riccati equation technique," International Journal of Non-Linear Mechanics, vol. 46, no. 5, pp. 758-771, 2011.

[27] C. Arduini and P. Baiocco, "Active magnetic damping attitude control for gravity gradient stabilized spacecraft," Journal of Guidance, Control, and Dynamics, vol. 20, no. 1, pp. 117-122, 1997.

[28] C. J. Damaren, "Comments on "Fully magnetic attitude control for spacecraft subject to gravity gradient"', Automatica, vol. 38, no. 12, p. 2189, 2002.

[29] M. Lovera and A. Astolfi, "Global attitude regulation using magnetic control," in Proceedings of the 40th IEEE Conference on Decision and Control (CDC '01), pp. 4604-4609, Orlando, FL, USA, December 2001.

[30] M. Lovera and A. Astolfi, "Spacecraft attitude control using magnetic actuators," Automatica, vol. 40, no. 8, pp. 1405-1414, 2004.

[31] M. Lovera and A. Astolfi, "Global magnetic attitude control of inertially pointing spacecraft," Journal of Guidance, Control, and Dynamics, vol. 28, no. 5, pp. 1065-1067, 2005.

[32] M. Lovera and A. Astolfi, "Global magnetic attitude control of spacecraft in the presence of gravity gradient," IEEE Transactions on Aerospace and Electronic Systems, vol. 42, no. 3, pp. 796-805, 2006.

[33] V. Bushenkov and G. Smirnov, Stabilization Problems with Constraints: Analysis and Computational Aspects, Gordon and Breach Science Publishers, Amsterdam, The Netherlands, 1997.

[34] L. S. Pontryagin, "Linear differential games. I," Soviet Mathematics. Doklady, vol. 8, pp. 769-771, 1967.

[35] R. N. Izmailov, "The peak effect in stationary linear systems with scalar inputs and outputs," Automation and Remote Control, vol. 48, no. 8, pp. 1018-1024, 1987.

[36] H. J. Sussmann and P. V. Kokotovic, "The peaking phenomenon and the global stabilization of nonlinear systems," IEEE Transactions on Automatic Control, vol. 36, no. 4, pp. 424-440, 1991.

[37] G. Smirnov, V. Bushenkov, and F. Miranda, "Advances on the transient growth quantification in linear control systems," International Journal of Applied Mathematics and Statistics, vol. 14, no. J09, pp. 82-92, 2009.

[38] S. P. Bhat, "Controllability of nonlinear time-varying systems: applications to spacecraft attitude control using magnetic actuation," IEEE Transactions on Automatic Control, vol. 50, no. 11, pp. 1725-1735, 2005.

[39] G. Smirnov, Introduction to the Theory of Differential Inclusions, American Mathematical Society, Providence, RI, USA, 2002.

[40] I. V. Burkov, "Asymptotic stabilization of the position of a rigid body with fixed point without velocity measurements," Systems and Control Letters, vol. 25, no. 3, pp. 205-209, 1995.

[41] S. Sastry, Nonlinear Systems: Analysis, Stability, and Control, Interdisciplinary Applied Mathematics, Vol. 10, Springer, Berlin, Germany, 1999. 

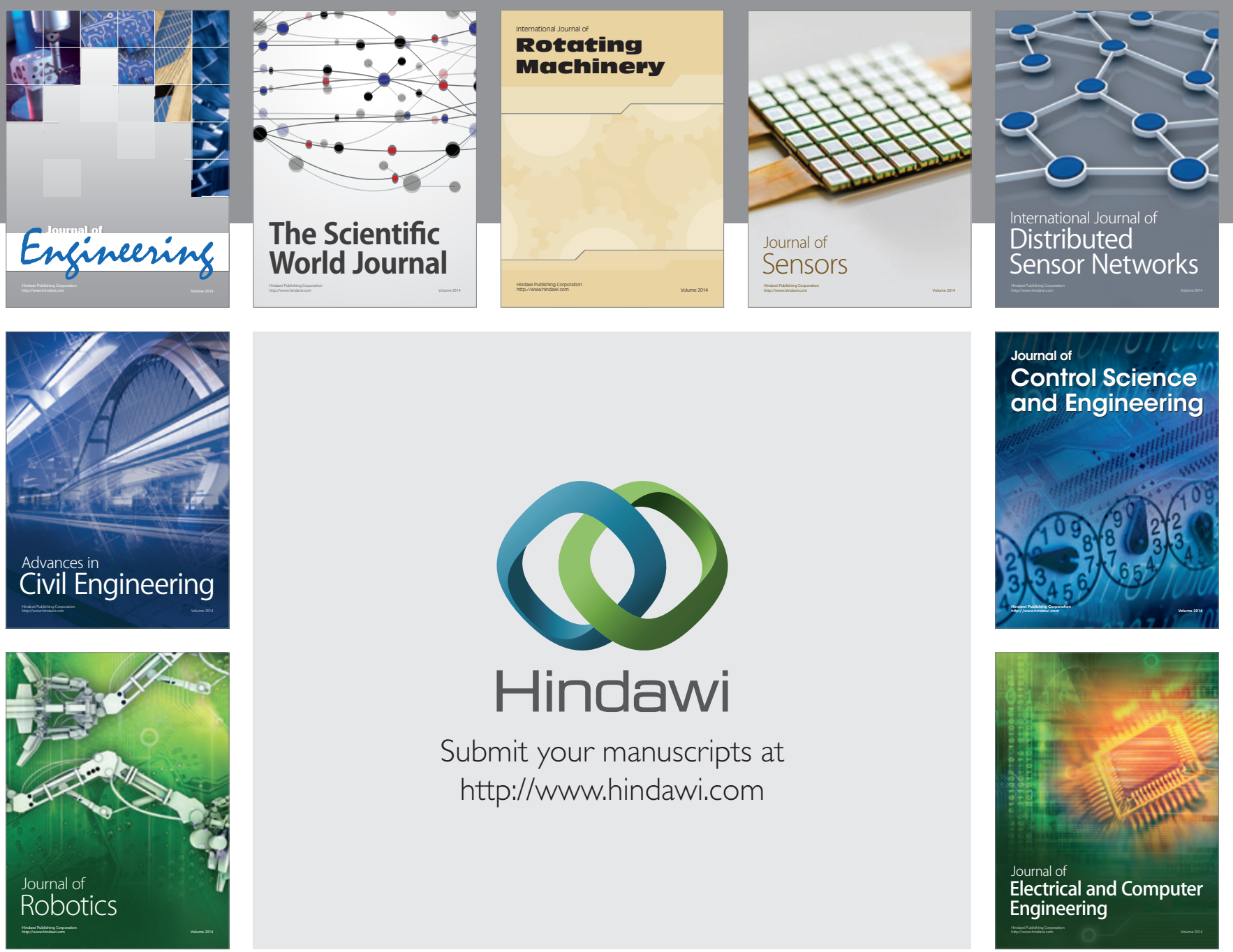

Submit your manuscripts at

http://www.hindawi.com
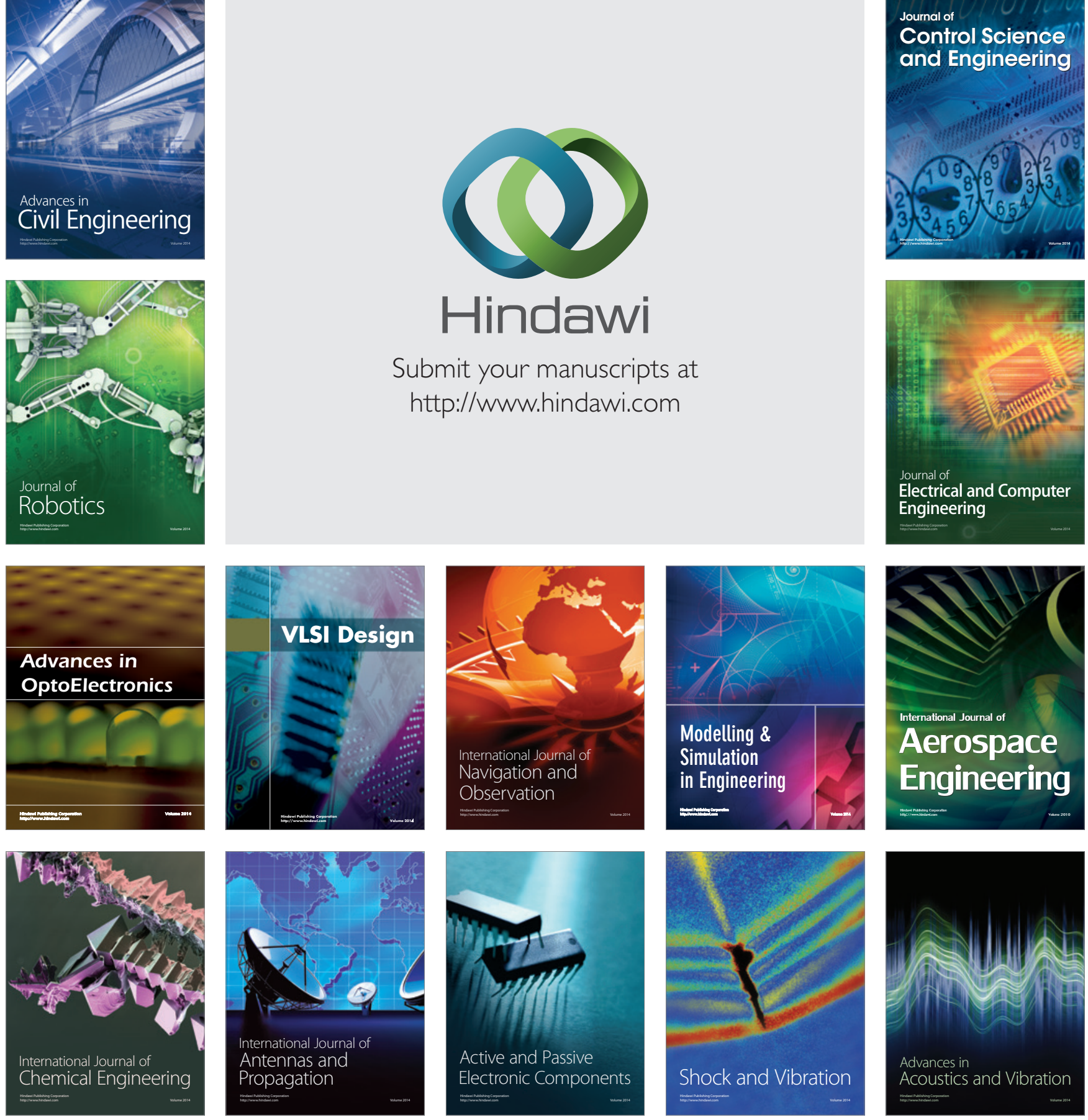